\title{
Regularization uncertainty in density models estimated from normal mode data
}

\author{
Joseph S. Resovsky and Michael H. Ritzwoller \\ University of Colorado at Boulder
}

\begin{abstract}
Normal mode structure coefficients provide important constraints on the long-wavelength component of 3-D mantle density $(\rho)$ structure, but inversions for independent models of $v_{s}, v_{p}$, and $\rho$ using normal mode data alone are ill-posed even at long wavelengths. Ill-posed inversions typically are regularized by imposing a priori assumptions on the set of estimated models, but such regularization can introduce important uncertainties in the models. We characterize these uncertainties for $\rho$ models estimated from current normal mode data using a set of 512 different "regularization schemes". These schemes sample a variety of plausible a priori assumptions about the nature and distribution of mantle heterogeneity by specifying allowable $v_{s}$, $v_{p}, \rho$, and boundary topography structures. The estimated $\rho$ models are fairly robust with respect to prior constraints on $v_{s}, v_{p}$, and topography. However, the character and amplitude of the estimated $\rho$ models depend strongly on how $\rho$ is allowed to decorrelate from $v_{s}$, and we display several models in which $\rho$ and $v_{s}$ decorrelate in very different depth intervals. Because these models all result from plausible prior constraints and fit the data equally and acceptably well, inversions of current normal mode data cannot robustly locate the decorrelation of $\rho$ from $v_{s}$. It remains possible that reliable $\rho$ models may be obtained in the future as more normal mode measurements are introduced to break the strong tradeoffs between upper and lower mantle $\rho$ structures that characterize current inversions.
\end{abstract}

\section{Introduction}

The estimation of reliable $3 \mathrm{D}$ mantle density models is among the most important and elusive objectives of global seismology. Independent models of $v_{s}, v_{p}$, and $\rho$ are essential to distinguish between chemical and thermal heterogeneity, and a well resolved image of density variations in the mantle is integral to improved geodynamic modeling.

Unfortunately, most seismic data are characterized by strong tradeoffs between sensitivity to $v_{s}$ heterogeneity and to all other forms of mantle structure [e.g., Lavely et al., 1994]. For this reason, inversions for seismic mantle models usually constrain lateral $\rho$ and $v_{p}$ heterogeneities to be perfectly correlated with $v_{s}$ structure at any depth. Such models employ depth-dependent multiplicative scaling factors to obtain $v_{p}$ and $\rho$ models from $v_{s}$ [e.g., Liu and Dziewonski, 1994; Masters, et al., 1996; Li and Romanowicz, 1996]. We refer to these as "scaling relation" models. New body-wave and surface-wave inversions have begun to produce reliable models of $v_{p}$ [Bolton, 1996; Vasco and Johnson, 1998] and

Copyright 1999 by the American Geophysical Union.

Paper number 1999GL900540.

0094-8276/99/1999GL900540\$05.00 bulk sound [e.g., Kennet et al., 1998], while surface-wave inversions for independent models of $v_{s}$ and $\rho$ [Tanimoto, 1991] have had more limited success.

Recent analyses have produced new catalogues of normal mode structure coefficients [e.g., Tromp and Zanzerkia, 1995; He and Tromp, 1996; Resovsky and Ritzwoller, 1998]. At long wavelengths, and particularly in the upper-mantle transition zone and the mid-mantle $(\sim 700-2000 \mathrm{~km})$, these measurements complement the sensitivity of body-wave and surface-wave data. Misfit statistics to normal mode data help to discriminate among recent models [Resovsky and Ritzwoller, 1998; hereafter R\&R98], and inversions of these data have produced a new long-wavelength scaling-relation mantle model [Resovsky and Ritzwoller, 1999; hereafter R\&R99]. There have also emerged models that exploit normal mode sensitivity to $v_{p}, \rho$, and topography on major radial discontinuities (hereafter called boundary topography) throughout the mantle [Ishii and Tromp, 1997; Tromp and Ishii, 1998; Kuo et al., 1998].

We have reported elsewhere [e.g., Resovsky and Ritzwoller, 1996], however, that there are strong tradeoffs in the inversion of normal mode data between structures of different kinds at different depths. The application of a priori constraints on the set of allowed models regularizes the inversion and helps to reduce these tradeoffs, but also has a significant impact on the character of $\rho$ models that emerge from the inversion. This impact is not quantified by standard resolution analyses [Trampert, 1998].

Therefore, the purpose of this letter is to characterize the sensitivity of $\rho$ models to regularization schemes based on a representative sampling of plausible a priori assumptions about allowed structures in the mantle.

\section{Defining regularization uncertainties}

We estimate a seismic Earth model $m$ by minimizing a cost function of the form $C_{\lambda}=\Delta_{D}(d, \mathbf{A} m)+\lambda \Delta_{M}\left(m, m_{0}\right)$, where the data, $d$, are a set of observed perturbations to the predictions of some initial model, the matrix $\mathbf{A}$ represents the (linearized) dependence of the normal mode data on Earth structures, $\lambda$ is an ad hoc damping constant, and $\Delta_{D}$ and $\Delta_{M}$ are metrics measuring the distance between observed and predicted data and between $m$ and a reference model $m_{0}$. These metrics can be assigned the forms $\Delta_{D}=$ $(d-\mathbf{A} m)^{\dagger} \mathbf{C}_{D}^{-1}(d-\mathbf{A} m)$ and $\Delta_{M}=\left(m-m_{0}\right)^{\dagger} \mathbf{C}_{M}^{-1}\left(m-m_{0}\right)$, where $\mathbf{C}_{D}$ is a covariance operator describing data errors and $\mathbf{C}_{M}$ is a covariance operator composed of a priori constraints on the model. We refer to the choice of $\lambda$ as "damping" and the choice of $m_{0}$ and $\mathbf{C}_{M}$ ("parameterization" in some literature) as "regularization". Because normal mode inversions are typically performed independently at each spherical harmonic degree and order, damping and regularization may vary at different degrees and orders. 


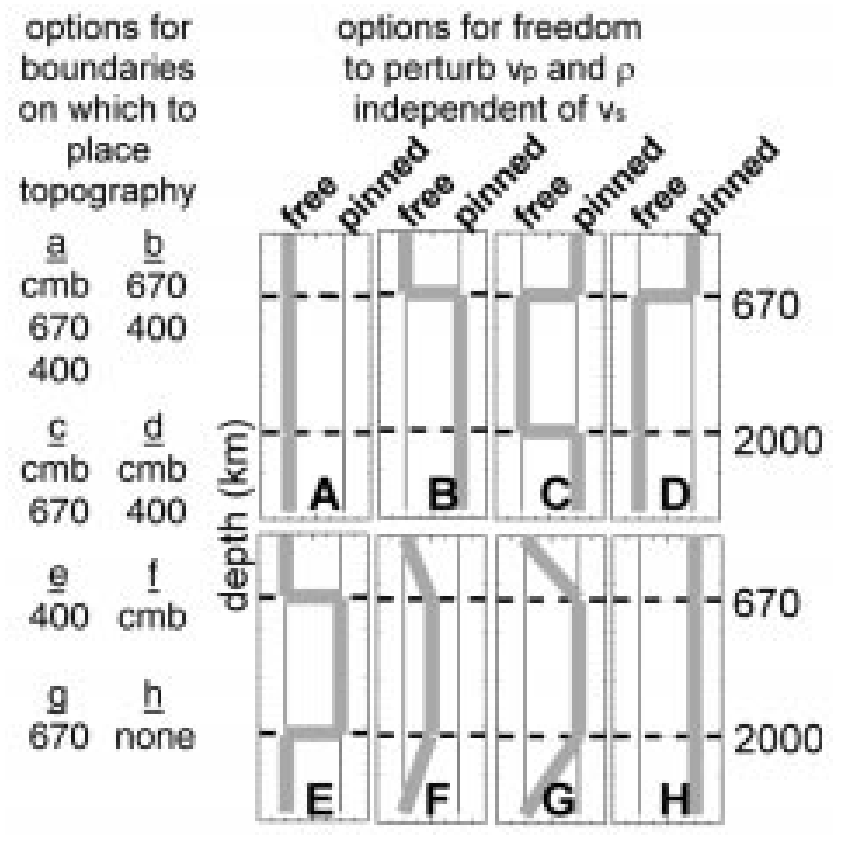

Figure 1. Menu for selecting and naming regularization schemes. For constraint function selections $A, B, C, D, E$, and $H, v_{p}$ and $\rho$ models are either "pinned" to scale with the $v_{s}$ model or "free" to become decorrelated from $v_{s}$ at some depths. Constraints $F$ and $G$ allow more decorrelation at the top and bottom of the mantle than at intermediate depths. Selections $a-h$ indicate combinations of boundaries on which topography is allowed. Regularization schemes and associated models are named such that, for example, label $\mathrm{ABc}$ implies $v_{p}$ selection $A, \rho$ selection selection $B$, and topography selection $c$.

It has rightly been reported [Ishii and Tromp, 1997; Tromp and Ishii, 1998] that straightforward choices of damping and regularization (i.e., $\lambda$ independent of spherical harmonic degree and order, uniform prior $-\mathbf{C}_{M}=\mathbf{I}$ ) can yield significant improvements in misfit to normal data and produce long-wavelength models of $\rho$ with reasonable amplitude and radial resolution. Resolution analyses, however, understate model uncertainties when there is significant uncertainty in the choice of regularization [Trampert, 1998]. Because we and others [Ishii and Tromp, 1997; Tromp and Ishii, 1998] have found that normal mode inversions are relatively robust with respect to the choice of the reference $m_{0}$, this letter examines the dependence of the estimated $\rho$ models on prior constraints encoded in $\mathbf{C}_{M}$. We refer to this as "regularization uncertainty".

\section{Regularization: Sampling plausible priors}

We start all of the inversions with the normal mode "scaling relation" model MM2_L12D8 of R\&R99, which is a 3-D $v_{s}$ model obtained by inverting normal mode structure coefficients from R\&R98, in which $v_{p}$ and $\rho$ scale with $v_{s}$ and there is no topography on any internal boundaries. The inversions of this letter further improve the fit to the normal mode data by perturbing $v_{s}, v_{p}, \rho$, and boundary components of MM2_L12D8. In these inversions structure is laterally parameterized with spherical harmonics through degree

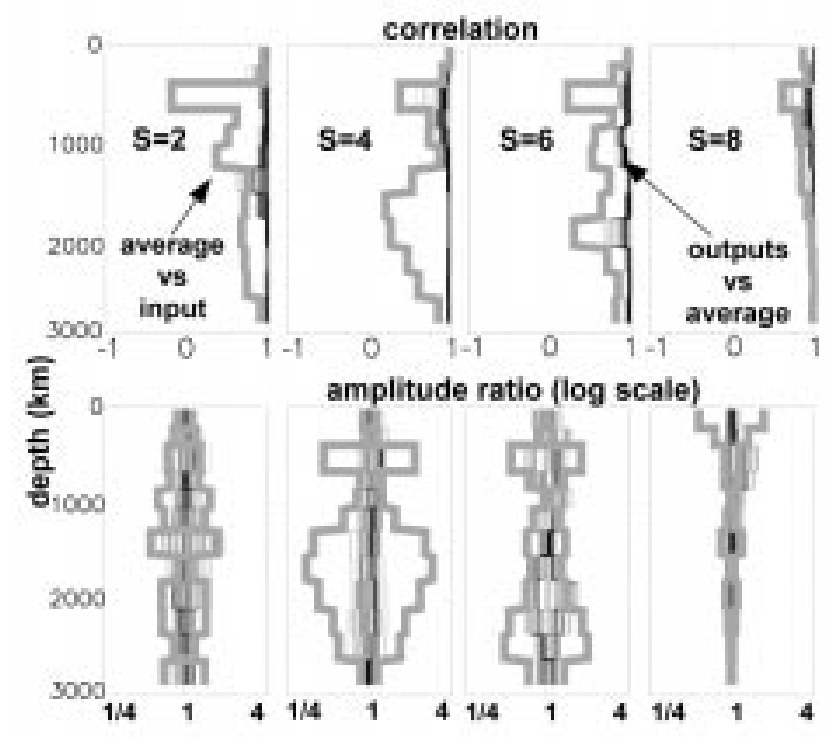

Figure 2. Geometry and amplitude ranges of $\rho$ models from a suite of inversions that employ $\rho$ constraint $A$ (Figure 1), together with 64 different $v_{p}$ and topography constraints. The thin black lines show the correlation of each model with their average (top) and the ratio of the RMS amplitude of each model to that to the average model (bottom), in each of the 12 model layers and at each structural degree. For comparison, the thick gray lines show the correlation of the average output model with the starting model, and the ratio of RMS amplitude of the average model to that of the input RMS amplitude. The inverse of the average amplitude ratio is also shown. Average perturbations to the $\rho$ model almost everywhere bound the range of perturbations around the average. $90 \%$ correlation confidence levels are at $(0.73,0.55,0.45,0.39)$ for degrees $(2,4,6,8)$. This shows that estimated $\rho$ models are all similar to one another and quite different from the input model. Thus $\rho$ estimates are relatively insensitive to prior constraints on $v_{p}$ and internal boundary topography.

and order 8. Topography is permitted on the $400 \mathrm{~km}, 670 \mathrm{~km}$, and $\mathrm{CMB}$ discontinuities, and the radial parameterization of volumetric perturbations is given by 12 layers as in R\&R99.

Figure 1 presents the menu from which we select 512 a priori "regularization schemes", using eight possible radial functions for constraining $v_{p}$ and $\rho$ perturbations and eight combinations of boundaries on which to allow topography. Where $v_{p}$ and $\rho$ are "pinned" to scale with $v_{s}$, they can be perturbed only through perturbations to $v_{s}$. We use $\left[\left(d \ln v_{p} / d \ln v_{s}\right),\left(d \ln \rho / d \ln v_{s}\right)\right]=[0.8,0.3]$ in the upper mantle and $[0.5,0.2]$ in the lower mantle, and the $v_{s}$ model is constrained to stay very near MM2_L12D8. Separate inversions are performed at each structural degree and all inversions are damped to ensure that output models meet specified size and stability conditions. We also performed inversions using geoid data together with the normal mode measurements, but, owing to persistent tradeoffs between internal boundary topography and $\rho$ structure, these inversions gave the same results as those discussed below. Odd degree results are not discussed because these inversions use fewer data and require damping strong enough to dominate expected regularization effects. 


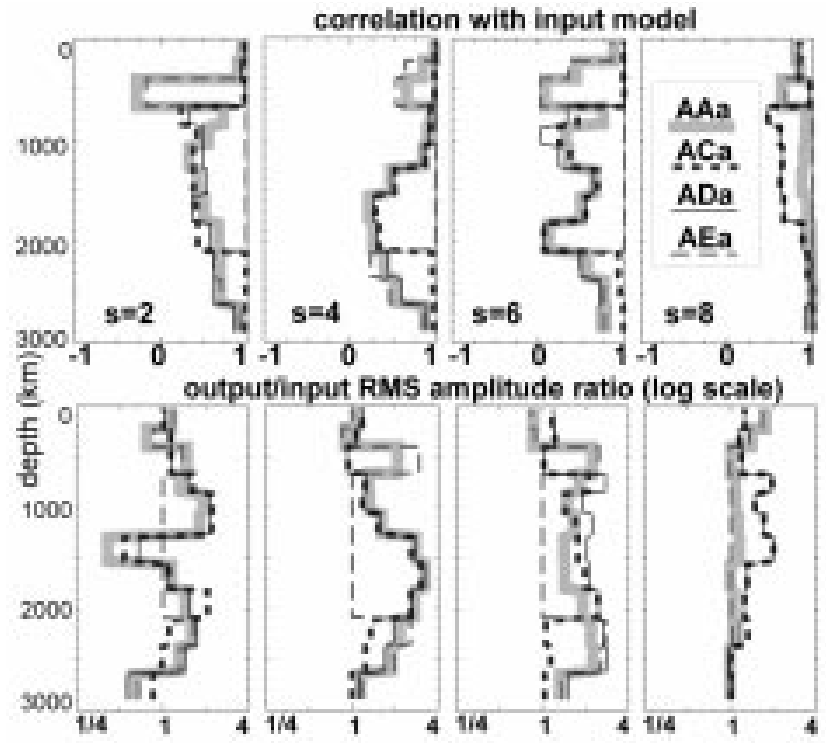

Figure 3. Profiles of $\rho$ models from inversions with four different $\rho$ regularizations, all with acceptable data fits. At each degree and depth for each model we show the correlation with the input $v_{s}$ model and the ratio of output and input RMS model amplitudes. Models $A C a, A D a$, and $A E a$ have been constrained to remain correlated with the output $v_{s}$ model in certain depth ranges (see Figure 1). The output $v_{s}$ model is slightly different from the input model. This shows that the estimated $\rho$ models can differ substantially from one another and still provide maximal improvement in fit to the data.

We believe that our suite of regularizations reflects a realistic variety of plausible a priori assumptions. The amplitude of boundary topography in the upper mantle has differed by as much as $50 \%$ in recent models [Flanagan and Shearer, 1998; Gu et al., 1998], and different assumptions about mantle viscosity in dynamic models can imply widely varying models of any kind of dynamic topography [e.g., Keiffer and Kellogg, 1998]. Where structural heterogeneity is dominantly thermal in origin, $v_{p}$ and $\rho$ should be highly correlated with $v_{s}$, while, in the presence of chemical heterogeneity, either $v_{p}$ or $\rho$ or both may be largely decorrelated from $v_{s}$. A blend of chemical and thermal heterogeneity is expected in the uppermost mantle, but may also be important in the transition zone and in the lowermost mantle [ Kellogg et al., 1999]. Therefore, decorrelations of either $v_{p}$ or $\rho$ from $v_{s}$ may be restricted to one or more of these regions or may be present throughout the mantle. The "priors" represented by the regularization schemes have been chosen to reflect these uncertainties in a variety of deep earth structures, particularly uncertainty in the depth intervals in which $\rho$ can decorrelate with $v_{s}$.

\section{Sensitivity of $\rho$ estimates to $v_{p}$ and boundary topography regularization}

Normal mode density models prove to be robust relative to the set of regularizing schemes for $v_{p}$ heterogeneity and boundary topography. That is, regularizing schemes that employ the same prior constraint on $\rho$ but varying constraints on $v_{p}$ and topography result in approximately con- sistent output $\rho$ models. Figure 2 exemplifies this by displaying the estimated $\rho$ models from the subset of 64 inversions that employ $\rho$ damping scheme $A$. The output models are all similar to one another and quite different from the input model.

This behavior is observed for each of the 8 sets of 64 models associated with particular prior constraints on $\rho$. From these observations, we infer that normal mode density models are fairly robust with respect to reasonable assumptions about $v_{p}$ and boundary topography.

\section{Sensitivity of $\rho$ estimates to $\rho$ regularization}

Reliable $\rho$ models from normal mode inversions must also be robust with respect to the full range of reasonable prior information about $\rho$ structure itself. We test the sensitivity to prior information about how $\rho$ is allowed to decorrelate from $v_{s}$ by considering subsets of the collection of $\rho$ models produced by regularization schemes that differ only in the $a$ priori radial functions that constrain $\rho$.

Figure 3 shows profiles of the density models resulting from four different regularization schemes, of which three restrict decorrelation of $\rho$ from $v_{s}$ to various limited depth ranges. Of our 512 models, 450 improve upon the $\chi^{2}$ misfit of MM2_L12D8 to the normal mode data by $15 \%$ or more, but only 15 achieve the best improvements, of $\sim 24 \%$. The four models displayed in Figure 3 are all from the latter group. In each model, $\rho$ decorrelates from $v_{s}$ in different depth ranges, which indicates important tradeoffs among various depths of decorrelation. For example, at degree 2, the model with decorrelation allowed only in the mid-mantle (ACa) displays a strong decorrelation in the potential slabpenetration zone at the top of the lower mantle. This replaces the decorrelation between $\rho$ and $v_{s}$ in $D^{\prime \prime}$ in the other models.

Because such different models result from plausible prior constraints and fit the data equally and acceptably well, the inversions cannot robustly locate the decorrelation of $\rho$ from $v_{s}$ at any particular depth.

\section{Summary and Conclusions}

We have demonstrated the significant effects of a priori constraints on normal mode $\rho$ inversions using simplified, but plausible, assumptions about the location and relative size of $v_{s}, v_{p}, \rho$ and topographic structure. We have observed (1) that varying the regularization of $v_{p}$ and topography produces $\rho$ models that are reasonably consistent with one another, but (2) that $\rho$ models are not similarly robust with respect to prior constraints that restrict decorrelation of $\rho$ from $v_{s}$ to different depth ranges.

It may appear that the second conclusion contradicts reports by other researchers [e.g., Tromp and Ishii, 1998] that density structure is relatively well resolved with normal mode data. To understand that these results are not at variance requires insight into the limitations of resolution analyses. A resolution estimate depends on a particular set of a priori assumptions. If uncertainties in a priori assumptions are large, resolution is not a good predictor of the reliability of the estimated structures. In inversions for 3-D density models using normal mode data, uncertainties in $a$ priori assumptions are so large as to render resolution estimates for any particular regularization scheme irrelevant. 
The reliability of future density models that result from normal mode data can be improved with the acquisition of structure coefficients from normal modes that have not yet been analyzed with recent normal mode data and methodologies. These data should yield improved models of upper mantle density structure, and, in turn, help to break the trade-offs between density structures at different depths that characterize current inversions. For example, in the most recent normal mode catalogues, all but a few modes along the ${ }_{2} S$ and ${ }_{0} T$ branches are sensitive to density in both the upper and lower mantle. The fundamental modes above $3 \mathrm{mHz}$ have not yet been subjected to recently developed normal mode analysis methods. More refined analyses of these modes may provide the exclusive sensitivity to upper mantle $\rho$ needed to improve upon the present inversions.

Acknowledgments. We are very grateful to several anonymous reviewers who helped us to clarify the presentation of the results of the inversions. This research was supported by NSF grant EAR-9706188.

\section{References}

Bolton, H., Long period travel times and the structure of the mantle, Ph.D. thesis, U.C. San Diego, 1996.

Flanagan, M. P., and P. M. Shearer, Global mapping of topography on transition zone velocity discontinuities by stacking SS precursors, J. Geophys. Res., 103, 2673-2692, 1998.

Gu, Y., A. M. Dziewonski, and C. B. Agee, Global decorrelation of the topography of transition zone discontinuities, Earth Planet. Sci. Lett. 157, 57-67, 1998.

He, X., and J. Tromp, Normal mode constraints on the structure of the Earth, J. Geophys. Res., 101, 20,053-20,082, 1996.

Ishii, M., and J. Tromp, Three-dimensional modeling of mantle heterogeneity using normal-mode splitting function coefficients (abstract), Eos Trans. AGU, 78(46), Fall Meet. Suppl., F460, 1997.

Keiffer, W. S., and L. H. Kellogg, Geoid anomalies and dynamic topography from time-dependent spherical axisymmetric mantle convection, Phys. Earth Planet. Inter., 106, 237-256, 1998.

Kellogg, L. H., B. H. Hager, and R. D. van der Hilst, Compositional stratification in the deep mantle, Science, 283, 18811891, 1999.

Kennett, B. L. N., S. Widiyantoro, and R. D. van der Hilst, Joint seismic tomography for bulk sound and shear wave speed in the Earth's mantle, J. Geophys. Res., 103, 12,469-12,493, 1998

Kuo, C., J. Durek, and B. Romanowicz, Mantle heterogeneity inferred from normal mode spectra (abstract), Eos Trans. AGU, 79(45), Fall Meet. Suppl., F627, 1998.
Lavely, E.M., A. Rodgers, and M.H. Ritzwoller, Can the differential sensitivity of body wave, mantle wave, and normal mode data resolve the trade-off between transition zone structure and boundary topography?, Phys. Earth Planet. Inter., 86, 117-146, 1994.

Li, X.-D., and B. Romanowicz, Global mantle shear-velocity model developed using nonlinear asymptotic coupling theory, J. Geophys. Res., 101, 22,245-22,272, 1996.

Liu, X.-F., and A. M. Dziewonski, Improved resolution of the lower-most mantle shear wave velocity structure obtained using SKS-S data, Eos Trans. AGU, 75, Spring Meet. Suppl., S232$234,1994$.

Masters, G., S. Johnson, G. Laske, and H. Bolton, A shearvelocity model of the mantle, Phil. Trans. R. Soc. Lond. A, 354, 1385 - 1411, 1996.

Resovsky, J. S., and M. H. Ritzwoller, Assessing 3D mantle models with new normal mode observations, Eos Trans. AGU, 77, Fall Meet. Suppl., F483, 1996.

Resovsky, J. S., and M. H. Ritzwoller, New and refined constraints on three-dimensional Earth structure from normal modes below $3 \mathrm{mHz}$, J. Geophys. Res., 103, 783-810, 1998.

Resovsky, J. S., and M. H. Ritzwoller, A degree 8 mantle shear velocity model from normal mode observations below $3 \mathrm{mHz}$, J. Geophys. Res., 104, 100-110, 1999.

Tanimoto, T., Waveform inversion for three-dimensional density and S wave structure, J. Geophys. Res., 96, 8167-8189, 1991.

Trampert, J., Global seismic tomography: the inverse problem and beyond, Inverse Problems, 14, 371-385, 1998.

Tromp, J., and M. Ishii, Normal mode and free-air gravity constraints on the Large Scale Structure of the Mantle (abstract), Eos Trans. AGU, 79(45), Fall Meet. Suppl., F598, 1998.

Tromp, J., and E. Zanzerkia, Toroidal splitting observations from the great 1994 Bolivia and Kuril Islands earthquakes, using along-branch coupling approximations, Geophys. Res. Lett., 22, 2297-3000, 1995.

Vasco, D. W.,, and L. R. Johnson, Whole earth structure estimated from seismic arrival times, J. Geophys. Res., 103, 26332671, 1998.

J. S. Resovsky, and M. H. Ritzwoller Department of Physics, University of Colorado at Boulder, CO 80309-0390. (e-mail: resovsky@lemond.colorado.edu)

(Received January 29, 1999; revised May 12, 1999; accepted May 24, 1999.) 